Reprod. Nutr. Dévelop., 1982, 22 (5), 851-864.

\title{
Influence d'une augmentation de la température sur certains aspects du catabolisme azoté chez la truite arc-en-ciel (Salmo gairdneri Rich.)
}

\author{
Françoise VELLAS, J.-P. PARENT, \\ Ingrid BAHAMONDES, Martine CHARPENTEAU
}

Laboratoire d'Ecophysiologie des Poissons, Université Paul Sabatier,

38, rue des 36 Ponts, 31078 Toulouse Cedex, France.

Summary. Effect of temperature increase on some aspects of nitrogen catabolism in rainbow trout (Salmo gairdneri Rich.).

Rainbow trout acclimatized to $9{ }^{\circ} \mathrm{C}$ were subjected to a temperature increase (up to $17{ }^{\circ} \mathrm{C}$ ) for $16 \mathrm{hrs}$. During the period of acclimatization to $17^{\circ} \mathrm{C}$, we studied blood ammonia and urea and the hepatic activity of glutamate dehydrogenase, glutaminase, uricase and arginase.

The daily mean rates of blood ammonia and urea did not differ significantly at 9 and $17^{\circ} \mathrm{C}$. However, the pattern of these two parameters during the circadian cycle was not the same at $9{ }^{\circ} \mathrm{C}$ as after 23 days at $17^{\circ} \mathrm{C}$. The enzymatic activities rose after one day at $17^{\circ} \mathrm{C}$ and remained unchanged, except for arginase which showed perfect thermal compensation.

During the circadian cycle, there was some similitude between glutaminase activity and blood ammonia at $9{ }^{\circ} \mathrm{C}$ and after 23 days at $17^{\circ} \mathrm{C}$, as well as between arginase activity and blood urea.

\section{Introduction.}

Bien qu'un certain nombre de travaux ait été effectué sur l'influence de la température sur l'excrétion azotée des Téléostéens (Pora et Precup, 1960; Péquin et Serfaty, 1963 ; Savitz, 1969 ; Vellas, 1973; Ogino et al., 1973 ; Guérin-Ancey, 1976 ; Kaushik, 1980 b ; Jobling, 1981), chez ces Poissons, peu d'études furent consacrées aux effets du facteur thermique sur les mécanismes responsables de la formation des déchets azotés.

On sait que, chez les Téléostéens, les principaux produits finaux du catabolisme azoté sont l'ammoniaque et l'urée (Smith, 1929 ; Fromm, 1963). Ces deux composés sont synthétisés en majeure partie dans le foie, et, transportés par le sang, ils sont principalement excrétés au niveau des branchies (Smith, 1929 ; Péquin et Serfaty, 1963 ; Goldstein et al., 1964; Vellas et Serfaty, 1967). 
L'ammoniaque, d'après Péquin (1967) et Péquin et al. (1969), proviendrait de la dégradation de la glutamine qui interviendrait dans la détoxication ammoniacale. Cependant, d'après McBean et al. (1966) et Walton et Cowey (1977), l'ammoniaque serait formée à partir de l'acide glutamique, produit à partir de I'acide $\alpha$-cétoglutarique par des réactions de transamination. L'urée est engendrée par le catabolisme de l'acide urique et la dégradation de l'arginine tissulaire et alimentaire (Goldstein et Forster, 1965 ; Vellas et al., 1969 ; Cvancara, 1969 a, b, c ; Vellas, 1973).

Chez les Téléostéens, les travaux relatifs aux effets de la température sur l'ammoniogenèse et l'uréogenèse sont limités aux recherches des modifications de l'ammoniémie chez la Carpe (Péquin et Serfaty, 1963) et chez la Truite arc-enciel (Fauconneau et Luquet, 1979) et de l'activité des enzymes uricolytiques chez la Carpe (Vellas, 1965) et de l'arginase chez l'Orfe (Marinescu, 1973).

Dans cette étude, nous nous sommes proposés d'analyser l'influence d'une augmentation thermique sur les mécanismes de la biosynthèse ammoniacale et uréique chez la Truite arc-en-ciel et, dans ce but, nous avons suivi, durant l'acclimatation à la nouvelle température, l'évolution de l'ammoniémie, de l'urémie et des activités des enzymes hépatiques intervenant ou susceptibles d'intervenir dans la formation de l'ammoniaque et de l'urée excrétées (glutamate deshydrogénase, glutaminase, uricase et arginase).

\section{Matériel et méthodes.}

1. Matériel d'étude et conditions de stabulation. - Les truites utilisées proviennent d'une pisciculture de la région du Sud-Ouest ( $\left.{ }^{1}\right)$. Les poissons, d'un poids moyen de $173 \pm 17 \mathrm{~g}$, sont placés dans 6 bassins (une quarantaine d'animaux par bassin) d'une capacité de 450 litres, alimentés par l'eau de la ville $(160 \mathrm{I} / \mathrm{h})$, le taux d'oxygène dissous étant toujours proche de 100 p. 100 de saturation. L'éclairement correspond à la photopériode naturelle de l'époque d'expérimentation (décembre à février).

Après 4 semaines d'acclimatation à $9^{\circ} \mathrm{C}$, les animaux subissent, en $16 \mathrm{~h}$, une augmentation thermique jusqu'à $17^{\circ} \mathrm{C}$ et sont gardés environ 3 semaines (23 jours) à cette nouvelle température.

Les truites sont nourries, une fois par jour (heure du repas : $8 \mathrm{~h}$ ), avec de l'aliment commercial renfermant 45 p. 100 de protéines brutes, 7 p. 100 de lipides et 5 p. 100 de cellulose (indications du fabricant $\left({ }^{2}\right)$ ). La distribution de l'aliment, effectuée par petites quantités, est arrêtée dès que les animaux n'acceptent plus de nourriture.

2. Prélèvements. - Le sang est recueilli par ponction intracardiaque sur des animaux maintenus en immersion. Après la prise de sang, le poisson est pesé, sacrifié, puis le foie est prélevé et conservé dans l'azote liquide pour des analyses ultérieures.

(1) Pisciculture expérimentale de I'I.N.R.A., Donzacq, 40360 Pomarez.

(2) Aliment composé complet pour Truites. Grande Semoulerie de l'Ouest, 16160 GondPontouvre. 
Les prélèvements sont réalisés un jour à $9{ }^{\circ} \mathrm{C}$ (à la fin de la période d'acclimatation) et le $1^{e r}, 2^{e}, 5^{e}, 8^{e}$ et $23^{e}$ jour après l'élévation thermique. Au cours du nycthémère, ils sont effectués $1 \mathrm{~h}, 3 \mathrm{~h}, 6 \mathrm{~h}, 12 \mathrm{~h}$ et $24 \mathrm{~h}$ après le repas.

3. Méthodes analytiques.

Ammoniémie et urémie. - L'ammoniaque et l'urée sont dosées, immédiatement après le prélèvement sanguin, selon la méthode de microdiffusion de Conway (1962).

Activités enzymatiques. - Les déterminations sont effectuées à la température de vie des animaux (Bennemann, 1977 ; Warner et al., 1978) c'est-à-dire à $9{ }^{\circ} \mathrm{C}$ pour les truites acclimatées à ce niveau thermique et à $17^{\circ} \mathrm{C}$ pour les autres. De plus, pour la glutamate déshydrogénase $(3)$, dans le but de connaître l'activité en fonction de la température d'analyse, des mesures sont réalisées à $17^{\circ} \mathrm{C}$ chez les animaux acclimatés à $9^{\circ} \mathrm{C}$ et à $9^{\circ} \mathrm{C}$ chez les animaux maintenus 23 jours à $17^{\circ} \mathrm{C}$.

L'activité de la glutamate deshydrogénase (E.C. 1.4.1.3) (activité dans le sens de la libération de $\mathrm{NH}_{3}$ ) est évaluée suivant le principe de la méthode de Bernt et Bergmeyer (1974). Le foie est broyé à l'appareil Ultra-Turrax dans de l'eau distillée glacée $(50 \mathrm{mg} / \mathrm{ml})$ puis centrifugé, $20 \mathrm{~min}$, à $27000 \mathrm{~g}$. Vingt $\mu \mathrm{l} \mathrm{de}$ surnageant sont mélangés à un milieu renfermant du tampon glycocollehydrazine $(0,65 \mathrm{mM}$ de glycocolle, 0,52 $\mathrm{mM}$ d'hydrazine) $\mathrm{pH} 9,0,20 \mu \mathrm{M}$ de L-glutamate de sodium, 1,5 $\mu \mathrm{M}$ d'ADP et 1,42 $\mu \mathrm{M}$ de NAD (volume final : $1,5 \mathrm{ml}$ ). Le $\mathrm{NADH}$ formé par la dégradation enzymatique du glutamate est mesuré spectrophotométriquement, à $340 \mathrm{~nm}$, au cours des 5 premières minutes de réaction.

L'activité de la glutaminase phosphate dépendante (E.C. 3.5.1.2) (seule glutaminase mise en évidence chez les Téléostéens, Walton et Cowey, 1977) est déterminée selon la méthode de Curthoys et Lowry (1973). Le tissu hépatique est homogénéisé, à l'appareil de Potter-Elvehjem, dans du tampon Tris- $\mathrm{HCl}$ glacé, $\mathrm{pH} 8.6(100 \mathrm{mg} / \mathrm{ml})$ et $0,5 \mathrm{ml}$ d'homogénat est ajouté à un milieu contenant $87,5 \mu \mathrm{M}$ de tampon Tris- $\mathrm{HCl}, \mathrm{pH} 8,6,0,35 \mu \mathrm{M}$ d'EDTA, $100 \mu \mathrm{M}$ de phosphate dissodique et $35 \mu \mathrm{M}$ de L-glutamine (volume final: 1,75 $\mathrm{ml}$ ). Après $30 \mathrm{~min}$ d'incubation à la température d'expérience, la réaction est arrêtée par $\mathrm{HCl} 2 \mathrm{~N}$. Le milieu est alors centrifugé $10 \mathrm{~min}$ à $12000 \mathrm{~g}$ et $50 \mu \mathrm{l}$ de surnageant sont utilisés pour doser, selon la technique de Curthoys et Lowry, l'acide glutamique qui résulte de la dégradation de la glutamine.

L'activité de I'uricase (E.C. 1.7.3.3.) est testée en adoptant le principe de la méthode de Kalckar (1947) et Praetorius (1953). Le foie est homogénéisé à l'appa-

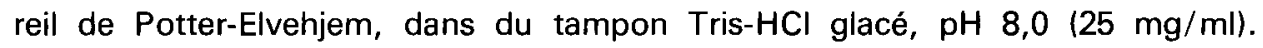
Un $\mathrm{ml}$ d'homogénat est mélangé à un milieu renfermant $1 \mathrm{mM}$ de tampon Tris- $\mathrm{HCl} \mathrm{pH} 9,35$ et 3,57 $\mu \mathrm{M}$ d'urate de sodium (volume final : 3,0 ml). Après $1 \mathrm{~h}$ d'expérience, la réaction est arrêtée et le milieu déféqué suivant le procédé décrit par Vellas (1973). L'acide urique non dégradé enzymatiquement est ensuite mesuré spectrophotométriquement à $284 \mathrm{~nm}$. Ion.

(3) Ce type d'expérimentation n'a pu être effectué pour les autres enzymes par défaut d'échantil- 
L'activité de l'arginase (E.C. 3.5.3.1) est évaluée suivant la méthode de Gilboe et Williams (1956 a, b). Un ml d'homogénat obtenu en broyant le foie, à l'appareil Ultra-Turrax, dans de l'eau distillée glacée $(50 \mathrm{mg} / \mathrm{ml})$, est ajouté à un milieu composé de $0,1 \mathrm{mM}$ de tampon pyrophosphate ajusté à $\mathrm{pH} 9,5$ et $8,61 \mu \mathrm{M}$ d'hydrochlorure de L-arginine (volume final : 5,0 ml). Après $10 \mathrm{~min}$, la réaction est arrêtée par de l'acide trichloracétique à 0,4 p. 100 et l'arginine non hydrolysée est déterminée par colorimétrie sur une partie aliquote du surnageant obtenu après centrifugation ( $5 \mathrm{~min}$ à $12000 \mathrm{~g}$ ).

Pour chaque activité enzymatique, les déterminations sont réalisées avec des témoins appropriés.

Protéines. - Le dosage des protéines est effectué suivant la méthode de Lowry et al. (1951).

4. Analyse statistique.

Les données sont traitées par une analyse de variance à deux facteurs: heure et jour, et le test de Bonferroni (Morrison, 1976) est utilisé pour déterminer les différences significatives entre les moyennes au seuil de probabilité de 5 et de 1 p. 100.

\section{Résultats.}

1. Croissance et prise de nourriture. - Le poids des animaux ne varie pas durant les 8 premiers jours d'acclimatation à $17^{\circ} \mathrm{C}$, mais le $23^{\mathrm{e}}$ jour à cette température, il y a un net accroissement pondéral (tabl. 1).

La prise de nourriture paraît diminuer les 2 premiers jours à $17^{\circ} \mathrm{C}$; toutefois, dès le $5^{\mathrm{e}}$ jour, elle est plus élevée qu'à $9^{\circ} \mathrm{C}$ et le $23^{\mathrm{e}}$ jour une forte augmentation est notée $(+128$ p. 100 par rapport à la consommation alimentaire à la température de $9^{\circ} \mathrm{C}$ ) (tabl. 1 ).

2. Rapport hépatosomatique et protéines hépatiques. - Le rapport hépatosomatique décroît le $5^{\mathrm{e}}$ jour d'adaptation thermique ; cependant, en fin d'expérimenta-

TABLE 1

Evolution du poids des animaux et de la prise de nourriture journalière durant l'acclimatation à $17{ }^{\circ} \mathrm{C}$

\begin{tabular}{lcccccc}
\hline Température & $9^{\circ} \mathrm{C}$ & \multicolumn{7}{c}{$17^{\circ} \mathrm{C}$} \\
\hline Jours d'expérience $\ldots \ldots$ & 1 & 2 & 5 & 8 & 23 \\
\hline Nombre d'animaux $\ldots \ldots$ & 45 & 30 & 30 & 30 & 30 & 30 \\
\hline Poids (g) $\ldots \ldots \ldots$ & $\begin{array}{r}226^{\mathrm{a}} \\
\pm 27\end{array}$ & $\begin{array}{r}218^{\mathrm{a}} \\
\pm 25\end{array}$ & $\begin{array}{r}215^{\mathrm{a}} \\
\pm 24\end{array}$ & $\begin{array}{r}230^{\mathrm{a}} \\
\pm 31\end{array}$ & $\begin{array}{r}248^{\mathrm{a}} \\
\pm 32\end{array}$ & $\begin{array}{r}312^{\mathrm{b}} \\
\pm 0\end{array}$ \\
\hline
\end{tabular}

\begin{tabular}{lllllll}
\hline $\begin{array}{l}\text { Prise de nourriture }(\mathrm{g} / \mathrm{kg} \\
\text { de poisson) } \ldots \ldots \ldots \ldots\end{array}$ & 15,3 & 12,1 & 13,0 & 20,9 & 27,8 & 33,4 \\
\hline
\end{tabular}

Valeur moyenne $\pm S D$. Les moyennes $n$ 'ayant pas le même indice sont significativement différentes $(P<0,01)$. 
tion, les valeurs obtenues ne sont pas différentes de celles que l'on observe chez les animaux acclimatés à $9^{\circ} \mathrm{C}$ (tabl. 2).

Quant aux protéines hépatiques, si elles diminuent progressivement durant les 5 premiers jours à $17^{\circ} \mathrm{C}$, le $23^{e}$ jour à ce niveau thermique, leur taux est plus élevé qu'à $9^{\circ} \mathrm{C}$ (tabl. 2).

3. Ammoniémie et urémie.

Ammoniémie. - Les valeurs des moyennes journalières de la concentration ammoniacale du sang ne sont pas différentes à $9^{\circ} \mathrm{C}$ et à $17^{\circ} \mathrm{C}$, aussi bien les premiers jours à cette dernière température que le $23^{e}$ jour (tabl. 3 ).

TABLEAU 2

Rapport hépatosomatique et protéines hépatiques chez la Truite acclimatée à $9{ }^{\circ} \mathrm{C}$ et durant l'acclimatation à $17{ }^{\circ} \mathrm{C}$

\begin{tabular}{|c|c|c|c|c|c|c|}
\hline Température & $9^{\circ} \mathrm{C}$ & & & $17^{\circ} \mathrm{C}$ & & \\
\hline Jours d'expérience ..... & & 1 & 2 & 5 & 8 & 23 \\
\hline $\begin{array}{l}\text { Rapport hépato-somati- } \\
\text { que (p. 100) } \ldots \ldots \ldots \ldots\end{array}$ & $\begin{array}{ll} & 1,31^{\mathrm{a}} \\
\pm & 0,25\end{array}$ & $\begin{array}{r}1,29^{a} \\
\pm 0,20\end{array}$ & $\begin{array}{l}1,29^{a} \\
\pm 0,23\end{array}$ & $\begin{array}{r}1,06^{c} \\
\pm 0,18\end{array}$ & $\begin{array}{l}1,25^{\mathrm{a}} \\
\pm 0,18\end{array}$ & $\begin{array}{r}1,36^{\mathrm{a}} \\
\pm 0,22\end{array}$ \\
\hline $\begin{array}{l}\text { Protéines hépatiques } \\
\text { (mg/animal de } 100 \mathrm{~g} \text { ) }\end{array}$ & $\begin{array}{r}160,20^{\mathrm{a}} \\
\pm \quad 31,54\end{array}$ & $\begin{array}{l}152,81^{\mathrm{ab}} \\
\pm 30,86\end{array}$ & $\begin{array}{l}146,35^{\mathrm{ab}} \\
\pm 28,20\end{array}$ & $\begin{array}{r}141,25^{b} \\
\pm 26,90\end{array}$ & $\begin{array}{l}148,60^{\mathrm{ab}} \\
\pm 24,26\end{array}$ & $\begin{array}{r}181,24^{\mathrm{C}} \\
\pm 26,20\end{array}$ \\
\hline
\end{tabular}

Valeur moyenne pour 30 à 45 individus $\pm S D$. Les movennes n'ayant pas le même indice sont significativement différentes $(a, b P<0,05 ; a$, $\mathbf{c}$ et $b, c P<0,01$ ).

\section{TABLEAU 3}

Evolution de l'ammoniémie, au cours du nycthémère, chez la Truite acclimatée à $9{ }^{\circ} \mathrm{C}$ et durant /'acclimatation à $17{ }^{\circ} \mathrm{C}(\mu \mathrm{g} \mathrm{N}$ ammoniacal $/ \mathrm{ml}$ de sang)

\begin{tabular}{|c|c|c|c|c|c|c|}
\hline \multirow{3}{*}{$\begin{array}{c}\text { Heures de prélèvement } \\
\text { après le repas }\end{array}$} & \multirow[t]{3}{*}{$9^{\circ} \mathrm{C}$} & \multicolumn{5}{|c|}{$17^{\circ} \mathrm{C}$} \\
\hline & & \multicolumn{5}{|c|}{ Jours d'expérience } \\
\hline & & 1 & 2 & 5 & 8 & 23 \\
\hline $1 \mathrm{~h}, \ldots \ldots \ldots \ldots \ldots \ldots$ & $\begin{array}{r}4,69 \\
+0,92\end{array}$ & $\begin{array}{r}4,24 \\
+0,78\end{array}$ & $\begin{array}{r}4,78 \\
\pm 0,94\end{array}$ & $\begin{array}{r}4,62 \\
\pm 1,10\end{array}$ & $\begin{array}{r}4,07 \\
\pm 1,15\end{array}$ & $\begin{array}{r}5,47 \\
+1,09\end{array}$ \\
\hline $3 \mathrm{~h}$ & $\begin{array}{r}7,11 \\
\pm 1,41\end{array}$ & $\begin{array}{r}5,95 \\
\pm 0,85\end{array}$ & $\begin{array}{r}5,10 \\
+0,92\end{array}$ & $\begin{array}{r}4,85 \\
\pm 1,37\end{array}$ & $\begin{array}{r}5,32 \\
+0,85\end{array}$ & $\begin{array}{r}6,21 \\
\pm 1,14\end{array}$ \\
\hline $6 \mathrm{~h} \ldots \ldots \ldots \ldots \ldots \ldots$ & $\begin{array}{r}7,72 \\
\pm \quad 1,71\end{array}$ & $\begin{array}{r}5,48 \\
\pm 0,86\end{array}$ & $\begin{array}{r}5,45 \\
\pm 0,56\end{array}$ & $\begin{array}{r}6,23 \\
+1,19\end{array}$ & $\begin{array}{r}6,30 \\
\pm \quad 1,70\end{array}$ & $\begin{array}{r}6,30 \\
\pm 0,85\end{array}$ \\
\hline $12 \mathrm{~h}$. & $\begin{array}{r}6,53 \\
\pm 0,76\end{array}$ & $\begin{array}{r}7,26 \\
+1,80\end{array}$ & $\begin{array}{r}6,61 \\
\pm 0,82\end{array}$ & $\begin{array}{r}7,72 \\
\pm 1,53\end{array}$ & $\begin{array}{r}8,10 \\
\pm 1,21\end{array}$ & $\begin{array}{r}7,65 \\
\pm 0,58\end{array}$ \\
\hline $24 \mathrm{~h} \ldots \ldots \ldots \ldots \ldots \ldots$ & $\begin{array}{r}5,30 \\
+\quad 1,50\end{array}$ & $\begin{array}{r}4,98 \\
+2,01\end{array}$ & $\begin{array}{r}3,36 \\
+0,27\end{array}$ & $\begin{array}{r}4,28 \\
+1,05\end{array}$ & $\begin{array}{r}4,87 \\
\pm 0,82\end{array}$ & $\begin{array}{r}4,25 \\
\pm 0,42\end{array}$ \\
\hline Moyenne journalière & $\begin{array}{r}6,24 \\
\pm 1,74\end{array}$ & $\begin{array}{r}5,58 \\
+1,63\end{array}$ & $\begin{array}{r}5,06 \\
\pm 1,27\end{array}$ & $\begin{array}{r}5,51 \\
\pm 1,76\end{array}$ & $\begin{array}{r}5,73 \\
+1,79\end{array}$ & $\begin{array}{r}5,91 \\
\pm 1,37\end{array}$ \\
\hline
\end{tabular}

Valeur moyenne pour 6 à 9 individus \pm SD. 
Au cours du nycthémère, l'évolution de l'ammoniémie apparaît, toutefois, modifiée par la température. A $9^{\circ} \mathrm{C}$, la valeur maximale est observée $6 \mathrm{~h}$ après le repas. A $17^{\circ} \mathrm{C}$, durant les 8 premiers jours d'acclimatation, si l'évolution de la teneur en ammoniaque du sang, de 1 à $6 \mathrm{~h}$ après le repas, est assez variable, le maximum est toujours atteint $12 \mathrm{~h}$ après l'alimentation, et, dans les heures qui suivent, il y a une baisse relativement importante de l'ammoniémie. Ces dernières modifications nycthémérales se retrouvent 23 jours après l'élévation thermique, et, à ce moment-là, on remarque, de plus, qu'il y a une augmentation de la concentration ammoniacale du sang $1 \mathrm{~h}$ après le repas (les variations obtenues $24 \mathrm{~h}$ et $1 \mathrm{~h}$ après le repas sont significativement différentes à $\mathrm{P}<0,05$ ).

Urémie. - Les moyennes journalières de l'urémie sont également identiques à 9 et $17^{\circ} \mathrm{C}$, excepté le $8^{e}$ jour d'acclimatation thermique où une diminution du taux uréique sanguin est notée (tabl. 4).

De même, comme pour l'ammoniémie, la concentration d'urée dans le sang atteint un maximum $6 \mathrm{~h}$ après le repas à $9^{\circ} \mathrm{C}$ et $12 \mathrm{~h}$ après le repas le $23^{\mathrm{e}}$ jour à $17^{\circ} \mathrm{C}$. Après le changement de température, on note, le 1er jour, les mêmes modifications nycthémérales qu'à $9^{\circ} \mathrm{C}$, mais dans les jours suivants $\left(2^{\mathrm{e}}, 5^{\mathrm{e}}\right.$ et $8^{e}$ jour) aucun parallélisme dans les variations observées ne peut être décelé.

4. Activités enzymatiques. - Dans le cas de la glutamate deshydrogénase, de la glutaminase et de l'uricase (tabl. 5, 6, 7), les valeurs des moyennes journalières de l'activité sont plus élevées à $17^{\circ} \mathrm{C}$ qu'à $9^{\circ} \mathrm{C}$. Cependant, la détermination, à

\section{TABLEAU 4}

Evolution de l'urémie, au cours du nycthémère, chez la Truite acclimatée à $9{ }^{\circ} \mathrm{C}$ et durant l'acclimatation à $17{ }^{\circ} \mathrm{C}$ ( $\mathrm{\mu g} \mathrm{N}$ uréique/ml de sang)

\begin{tabular}{|c|c|c|c|c|c|c|}
\hline \multirow{3}{*}{$\begin{array}{l}\text { Heures de prélèvement } \\
\text { après le repas }\end{array}$} & \multirow[t]{3}{*}{$9^{\circ} \mathrm{C}$} & \multicolumn{5}{|c|}{$17^{\circ} \mathrm{C}$} \\
\hline & & \multicolumn{5}{|c|}{ Jours d'expérience } \\
\hline & & 1 & 2 & 5 & 8 & 23 \\
\hline $1 \mathrm{~h} \ldots \ldots \ldots \ldots \ldots$ & $\begin{array}{r}11,67 \\
\pm 3,53\end{array}$ & $\begin{array}{r}12,28 \\
\pm \quad 2,46 \\
\end{array}$ & $\begin{array}{r}14,14 \\
\pm \quad 4,33\end{array}$ & $\begin{array}{r}12,08 \\
\pm \quad 5,09\end{array}$ & $\begin{array}{r}7,58 \\
+3,96\end{array}$ & $\begin{array}{r}8,98 \\
+\quad 1,76\end{array}$ \\
\hline $3 h \ldots \ldots \ldots \ldots \ldots$ & $\begin{array}{r}15,15 \\
\pm \quad 7,16\end{array}$ & $\begin{array}{r}18,09 \\
\pm 4,24\end{array}$ & $\begin{array}{r}17,78 \\
\pm \quad 3,81\end{array}$ & $\begin{array}{r}13,52 \\
\pm 8,45\end{array}$ & $\begin{array}{r}7,41 \\
\pm \quad 2,81\end{array}$ & $\begin{array}{r}10,51 \\
\pm 2,32\end{array}$ \\
\hline $6 \mathrm{~h} \ldots \ldots \ldots \ldots \ldots \ldots$ & $\begin{array}{r}15,33 \\
\pm \quad 1,04\end{array}$ & $\begin{array}{r}21,19 \\
\pm \quad 5,54\end{array}$ & $\begin{array}{r}15,61 \\
\pm \quad 3,36\end{array}$ & $\begin{array}{r}12,66 \\
\pm \quad 1,64\end{array}$ & $\begin{array}{r}10,41 \\
\pm 2,99\end{array}$ & $\begin{array}{r}12,88 \\
+\quad 3,44\end{array}$ \\
\hline $12 h \ldots \ldots \ldots \ldots \ldots$ & $\begin{array}{r}12,00 \\
\pm \quad 2,15\end{array}$ & $\begin{array}{r}15,75 \\
\pm \quad 6,48\end{array}$ & $\begin{array}{r}14,92 \\
\pm \quad 6,05\end{array}$ & $\begin{array}{r}15,85 \\
\pm \quad 4,60\end{array}$ & $\begin{array}{r}9,99 \\
+2,10\end{array}$ & $\begin{array}{r}18,18 \\
+\quad 3,05\end{array}$ \\
\hline $24 \mathrm{~h} \ldots \ldots \ldots \ldots \ldots$ & $\begin{array}{r}9,19 \\
\pm 3,51\end{array}$ & $\begin{array}{r}11,24 \\
\pm 5,51\end{array}$ & $\begin{array}{r}9,81 \\
\pm 3,64 \\
\end{array}$ & $\begin{array}{r}8,29 \\
\pm 3,04 \\
\end{array}$ & $\begin{array}{r}6,81 \\
+2,58\end{array}$ & $\begin{array}{r}11,04 \\
\pm \quad 3,97\end{array}$ \\
\hline Moyenne journalière .... & $\begin{array}{r}12,71 \\
\pm \quad 5,17\end{array}$ & $\begin{array}{r}15,71 \\
\pm \quad 5,85\end{array}$ & $\begin{array}{r}15,56 \\
\pm \quad 4,36\end{array}$ & $\begin{array}{r}12,44 \\
\pm \quad 5,21\end{array}$ & $\begin{array}{r}8,47 \\
+3,27\end{array}$ & $\begin{array}{r}12,34 \\
\pm \quad 4,06\end{array}$ \\
\hline
\end{tabular}

Valeur moyenne pour 6 à 9 individus $\pm S D$. 


\section{TABLEAU 5}

Activité de la glutamate deshydrogénase du foie, au cours du nycthémère, chez la Truite acclimatée à $9{ }^{\circ} \mathrm{C}$ et durant l'acclimatation à $17^{\circ} \mathrm{C}(\mu \mathrm{M}$ acide glutamique hydrolysé/min et /g de protéine)

\begin{tabular}{|c|c|c|c|c|c|c|}
\hline \multirow{3}{*}{$\begin{array}{l}\text { Heures de prélèvement } \\
\text { après le repas }\end{array}$} & \multirow[t]{3}{*}{$9^{\circ} \mathrm{C}$} & \multicolumn{5}{|c|}{$17^{\circ} \mathrm{C}$} \\
\hline & & \multicolumn{5}{|c|}{ Jours d'expérience } \\
\hline & & 1 & 2 & 5 & 8 & 23 \\
\hline $1 \mathrm{~h}$ & $\begin{array}{r}47,7 \\
\pm \quad 4,9\end{array}$ & $\begin{array}{r}76,0 \\
\pm \quad 9,9\end{array}$ & $\begin{array}{r}85,0 \\
\pm \quad 17,4\end{array}$ & $\begin{array}{r}85,8 \\
+\quad 6,5\end{array}$ & $\begin{array}{r}83,2 \\
\pm \quad 5,0\end{array}$ & $\begin{array}{r}74,3 \\
+15,8\end{array}$ \\
\hline $3 h \ldots \ldots \ldots \ldots \ldots$ & $\begin{array}{r}45,2 \\
+15,0\end{array}$ & $\begin{array}{r}82,3 \\
\pm \quad 5,1\end{array}$ & $\begin{array}{r}99,2 \\
\pm 26,9\end{array}$ & $\begin{array}{r}79,7 \\
\pm \quad 4,6\end{array}$ & $\begin{array}{r}87,0 \\
\pm 10,2\end{array}$ & $\begin{array}{r}76,1 \\
+15,4\end{array}$ \\
\hline $6 \mathrm{~h} \ldots \ldots \ldots$ & $\begin{array}{r}50,8 \\
+\quad 7,8\end{array}$ & $\begin{array}{r}74,9 \\
+\quad 17,2\end{array}$ & $\begin{array}{r}90,4 \\
+31,8\end{array}$ & $\begin{array}{r}71,9 \\
+11,6\end{array}$ & $\begin{array}{r}68,6 \\
+12,0\end{array}$ & $\begin{array}{r}70,8 \\
\pm \quad 11,2\end{array}$ \\
\hline $12 \mathrm{~h}$ & $\begin{array}{r}48,5 \\
\pm \quad 5,0\end{array}$ & $\begin{array}{r}74,1 \\
\pm \quad 7,8 \\
\end{array}$ & $\begin{array}{r}83,4 \\
\pm \quad 6,0\end{array}$ & $\begin{array}{r}83,4 \\
\pm 10,7\end{array}$ & $\begin{array}{r}81,5 \\
\pm 10,5\end{array}$ & $\begin{array}{r}73,0 \\
\pm 22,1\end{array}$ \\
\hline$\ldots \ldots \ldots \ldots \ldots \ldots$ & $\begin{array}{r}51,6 \\
\pm \quad 11,4 \\
\end{array}$ & $\begin{array}{r}61,4 \\
\pm \quad 7,0 \\
\end{array}$ & $\begin{array}{r}97,6 \\
\pm \quad 14,1 \\
\end{array}$ & $\begin{array}{r}85,5 \\
+22,5 \\
\end{array}$ & $\begin{array}{r}80,5 \\
\pm 10,9 \\
\end{array}$ & $\begin{array}{r}82,8 \\
\pm 13,8 \\
\end{array}$ \\
\hline Moyenne journalière & $\begin{array}{r}48,8^{a} \\
+\quad 9,4\end{array}$ & $\begin{aligned} & 73,6^{b} \\
\pm & 11,2\end{aligned}$ & $\begin{aligned} & 93,9^{c} \\
\pm & 18,8\end{aligned}$ & $\begin{aligned} & 81,2^{b c} \\
\pm & 12,5\end{aligned}$ & $\begin{aligned} & 80,1^{b c} \\
\pm & 11,0\end{aligned}$ & $\begin{array}{r}75,4^{b} \\
+14,8\end{array}$ \\
\hline
\end{tabular}

Valeur moyenne pour 6 individus \pm SD. Les moyennes n'ayant pas le même indice sont significativement différentes $(P<0,01)$.

TABLEAU 6

Activité glutaminasique du foie, au cours du nycthémère, chez la Truite acclimatée à $9{ }^{\circ} \mathrm{C}$ et durant l'acclimatation à $17{ }^{\circ} \mathrm{C}(\mu \mathrm{M}$ glutamine hydrolysée/h et $/ \mathrm{g}$ de protéine)

\begin{tabular}{|c|c|c|c|c|c|}
\hline Température & $9^{\circ} \mathrm{C}$ & \multicolumn{4}{|c|}{$17^{\circ} \mathrm{C}$} \\
\hline \multirow{2}{*}{$\begin{array}{l}\text { Heures de prélèvement } \\
\text { après le repas }\end{array}$} & & \multicolumn{4}{|c|}{ Jours d'expérience } \\
\hline & & 2 & 5 & 8 & 23 \\
\hline $1 \mathrm{~h}$ & $\begin{array}{r}187,4 \\
+\quad 60,8\end{array}$ & $\begin{array}{r}583,0 \\
+107,2\end{array}$ & $\begin{array}{r}398,4 \\
+\quad 149,0\end{array}$ & $\begin{array}{r}518,4 \\
+130,8\end{array}$ & $\begin{array}{r}381,6 \\
+\quad 93,8\end{array}$ \\
\hline $3 \mathrm{~h}$ & $\begin{array}{r}234,8 \\
\pm \quad 78,0\end{array}$ & $\begin{array}{r}510,6 \\
+\quad 858\end{array}$ & $\begin{array}{r}428,4 \\
\pm 117,2 \\
\end{array}$ & $\begin{array}{r}386,4 \\
+172,4\end{array}$ & $\begin{array}{r}438,0 \\
+\quad 73,2\end{array}$ \\
\hline $6 \mathrm{~h}$ & $\begin{array}{r}281,4 \\
+\quad 58,8\end{array}$ & $\begin{array}{r}336,4 \\
+135,6\end{array}$ & $\begin{array}{r}447,4 \\
\pm 106,0\end{array}$ & $\begin{array}{r}548,4 \\
\pm 134,4\end{array}$ & $\begin{array}{r}441,5 \\
\pm \quad 96,9\end{array}$ \\
\hline $12 \mathrm{~h}$. & $\begin{array}{r}353,2 \\
+\quad 128,4\end{array}$ & $\begin{array}{r}461,4 \\
+\quad 74,8\end{array}$ & $\begin{array}{r}602,4 \\
\pm \quad 69,2\end{array}$ & $\begin{array}{r}477,0 \\
\pm 136,2\end{array}$ & $\begin{array}{r}668,8 \\
\pm 149,6\end{array}$ \\
\hline $24 \mathrm{~h}$ & $\begin{array}{r}187,4 \\
+\quad 59,2\end{array}$ & $\begin{array}{r}620,0 \\
+198,0\end{array}$ & $\begin{array}{r}465,0 \\
+135,8\end{array}$ & $\begin{array}{r}442,4 \\
+\quad 122,4\end{array}$ & $\begin{array}{r}400,0 \\
+122,8\end{array}$ \\
\hline Moyenne journalière & $\begin{array}{r}248,8^{a} \\
\pm \quad 96,8\end{array}$ & $\begin{aligned} & 506,4^{\mathrm{b}} \\
\pm & 180,0\end{aligned}$ & $\begin{aligned} & 468,4^{b} \\
\pm & 128,0\end{aligned}$ & $\begin{array}{l}\quad 474,6^{\mathrm{b}} \\
\pm \quad 125,4\end{array}$ & $\begin{aligned} & 475,6^{b} \\
+ & 151,7\end{aligned}$ \\
\hline
\end{tabular}

Valeur moyenne pour 6 individus \pm SD. Les moyennes n'ayant pas le même indice sont signifi. cativement différentes $(\mathrm{P}<0,01)$. 


\section{TABLEAU 7}

Activité uricasique du foie, au cours du nycthémère, chez la Truite acclimatée à $9{ }^{\circ} \mathrm{C}$ et durant /'acclimatation à $17^{\circ} \mathrm{C}(\mu \mathrm{M}$ acide urique hydrolysé/h et /g de protéine)

\begin{tabular}{|c|c|c|c|c|c|c|}
\hline Température & $9^{\circ} \mathrm{C}$ & & & $17^{\circ} \mathrm{C}$ & & \\
\hline \multirow{2}{*}{$\begin{array}{l}\text { Heures de prélèvement } \\
\text { après le repas }\end{array}$} & & \multicolumn{5}{|c|}{ Jours d'expérience } \\
\hline & & 1 & 2 & 5 & 8 & 23 \\
\hline $1 \mathrm{~h}$. & $\begin{array}{r}309 \\
\pm 102\end{array}$ & $\begin{array}{r}595 \\
+188\end{array}$ & $\begin{array}{r}706 \\
\pm 172\end{array}$ & $\begin{array}{r}486 \\
+199\end{array}$ & $\begin{array}{r}805 \\
+117\end{array}$ & $\begin{array}{r}467 \\
+100\end{array}$ \\
\hline $3 \mathrm{~h}$ & $\begin{array}{r}345 \\
\pm 148\end{array}$ & $\begin{array}{r}649 \\
\pm 283\end{array}$ & $\begin{array}{r}477 \\
\pm 130\end{array}$ & $\begin{array}{r}535 \\
\pm \quad 294\end{array}$ & $\begin{array}{r}687 \\
\pm 352\end{array}$ & $\begin{array}{r}474 \\
\pm 133\end{array}$ \\
\hline $6 \mathrm{~h}$. & $\begin{array}{r}334 \\
+\quad 94\end{array}$ & $\begin{array}{r}608 \\
\pm \quad 174\end{array}$ & $\begin{array}{r}589 \\
+268\end{array}$ & $\begin{array}{r}744 \\
+106\end{array}$ & $\begin{array}{r}730 \\
+150\end{array}$ & $\begin{array}{r}795 \\
\pm 290\end{array}$ \\
\hline$\ldots \ldots \ldots \ldots \ldots$ & $\begin{array}{r}365 \\
\pm 163\end{array}$ & $\begin{array}{r}741 \\
+\quad 48\end{array}$ & $\begin{array}{r}697 \\
+119\end{array}$ & $\begin{array}{r}644 \\
\pm 261\end{array}$ & $\begin{array}{r}838 \\
\pm \quad 181\end{array}$ & $\begin{array}{r}651 \\
\pm 223\end{array}$ \\
\hline $24 \mathrm{~h}$ & $\begin{array}{r}283 \\
\pm \quad 44\end{array}$ & $\begin{array}{r}611 \\
\pm 290\end{array}$ & $\begin{array}{r}530 \\
\pm \quad 192\end{array}$ & $\begin{array}{r}391 \\
\pm \quad 172\end{array}$ & $\begin{array}{r}700 \\
\pm 240\end{array}$ & $\begin{array}{r}447 \\
\pm \quad 105\end{array}$ \\
\hline Moyenne journalière .... & $\begin{aligned} & 326^{a} \\
\pm & 110\end{aligned}$ & 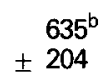 & $\begin{aligned} & 596^{b} \\
\pm & 189\end{aligned}$ & $\begin{aligned} & 560^{b} \\
\pm & 230\end{aligned}$ & $\begin{aligned} & 752^{c} \\
+ & 208\end{aligned}$ & $\begin{aligned} & 562^{b} \\
+ & 214\end{aligned}$ \\
\hline
\end{tabular}

Valeur moyenne pour 6 individus \pm SD. Les moyennes n'ayant pas le même indice sont significativement différentes $(\mathrm{P}<0,01)$.

la même température d'analyse, de l'activité de la glutamate deshydrogénase chez les animaux acclimatés à $9^{\circ} \mathrm{C}$ et chez les animaux maintenus 23 jours à $17{ }^{\circ} \mathrm{C}, \mathrm{n}^{\prime}$ indique pas de variations entre les deux lots de Poissons (tabl. 8). Dans le cas de l'arginase (tabl. 9), il y a un accroissement des moyennes journalières les 8 premiers jours après la hausse thermique, mais, le $23^{e}$ jour à $17^{\circ} \mathrm{C}$, on n'observe plus de différences avec l'activité des animaux acclimatés à $9^{\circ} \mathrm{C}$.

Les mesures effectuées au cours du nycthémère n'ont pas révélé de modifications de l'activité de la glutamate deshydrogénase et de l'uricase. Dans le cas de la glutaminase, à $9^{\circ} \mathrm{C}$, on note une augmentation de l'activité de 3 à $12 \mathrm{~h}$ après le repas ; à $17^{\circ} \mathrm{C}$, durant les premiers jours d'adaptation à cette température, les variations postprandiales sont assez diverses et, le $23^{e}$ jour, l'activité maximale est obervée $12 \mathrm{~h}$ après la prise de nourriture. Enfin, pour l'arginase, s'il n'y a pratiquement pas de modifications nycthémérales au cours des 8 premiers jours d'acclimatation thermique, on remarque, à $9{ }^{\circ} \mathrm{C}$ et le $23^{\mathrm{e}}$ jour à $17^{\circ} \mathrm{C}$, une évolution de l'activité enzymatique. Ainsi, l'activité arginasique est la plus élevée $6 \mathrm{~h}$ après le repas à $9^{\circ} \mathrm{C}$ et $12 \mathrm{~h}$ après le repas à $17^{\circ} \mathrm{C}$.

\section{Discussion.}

Fauconneau et Luquet (1979) ont signalé que, chez la Truite arc-en-ciel soumise à une élévation de température (dans les limites thermiques naturelles), il 


\section{TABLEAU 8}

Activité de la glutamate deshydrogénase en fonction de la température d'analyse ( $\mu \mathrm{M}$ acide glutamique hydrolysé/min et/g de protéine)

\begin{tabular}{crc}
\hline \multirow{2}{*}{$\begin{array}{c}\text { Température } \\
\text { d'acclimatation }\end{array}$} & \multicolumn{2}{c}{ Température d'analyse } \\
\cline { 2 - 3 } & $9{ }^{\circ} \mathrm{C}$ & $17{ }^{\circ} \mathrm{C}$ \\
\hline $9^{\circ} \mathrm{C}$ & 48,5 & 70,9 \\
& $\pm 5,0$ & $\pm 13,0$ \\
$17{ }^{\circ} \mathrm{C}$ & 47,5 & 73,0 \\
& $\pm 6,1$ & $\pm 22,1$ \\
\hline
\end{tabular}

Valeur moyenne pour 6 individus $\pm S D$. Echantillon prélevé $12 \mathrm{~h}$ après le repas.

\section{TABLEAU 9}

Activité arginasique du foie, au cours du nycthémère, chez la Truite acclimatée à $9{ }^{\circ} \mathrm{C}$ et durant l'acclimatation à $17{ }^{\circ} \mathrm{C}(\mu \mathrm{M}$ arginine hydrolysée $/ m i n$ et $/ \mathrm{g}$ de protéine)

\begin{tabular}{|c|c|c|c|c|c|c|}
\hline \multirow{3}{*}{$\begin{array}{l}\text { Heures de prélèvement } \\
\text { après le repas }\end{array}$} & \multirow[t]{3}{*}{$9^{\circ} \mathrm{C}$} & \multicolumn{5}{|c|}{$17^{\circ} \mathrm{C}$} \\
\hline & & \multicolumn{5}{|c|}{ Jours d'expérience } \\
\hline & & 1 & 2 & 5 & 8 & 23 \\
\hline $1 \mathrm{~h} \ldots \ldots \ldots \ldots \ldots \ldots$ & $\begin{array}{r}92,8 \\
\pm 16,2\end{array}$ & $\begin{array}{r}112,1 \\
\pm 22,4\end{array}$ & $\begin{array}{r}123,1 \\
\pm 14,7\end{array}$ & $\begin{array}{r}103,2 \\
\pm \quad 1,8\end{array}$ & $\begin{array}{r}107,1 \\
\pm \quad 11,1\end{array}$ & $\begin{array}{r}80,7 \\
\pm 14,4\end{array}$ \\
\hline $3 \mathrm{~h}$ & $\begin{array}{r}79,9 \\
+\quad 14,9\end{array}$ & $\begin{array}{r}128,8 \\
+\quad 14,4\end{array}$ & $\begin{array}{r}132,8 \\
\pm \quad 13,8\end{array}$ & $\begin{array}{r}108,5 \\
+24,6\end{array}$ & $\begin{array}{r}115,3 \\
\pm 40,7\end{array}$ & $\begin{array}{r}95,1 \\
+17,0\end{array}$ \\
\hline$\ldots \ldots \ldots \ldots \ldots$ & $\begin{array}{r}108,7 \\
\pm \quad 9,7\end{array}$ & $\begin{array}{r}116,9 \\
\pm \quad 12,8\end{array}$ & $\begin{array}{r}125,4 \\
+\quad 14,3\end{array}$ & $\begin{array}{r}98,6 \\
+11,2\end{array}$ & $\begin{array}{r}113,4 \\
\pm 24,9\end{array}$ & $\begin{array}{r}100,5 \\
+\quad 9,5\end{array}$ \\
\hline $12 \mathrm{~h} \ldots \ldots \ldots \ldots \ldots \ldots$ & $\begin{array}{r}88,3 \\
\pm \quad 6,3\end{array}$ & $\begin{array}{r}109,9 \\
\pm \quad 8,9\end{array}$ & $\begin{array}{r}124,4 \\
+\quad 16,6\end{array}$ & $\begin{array}{r}109,8 \\
\pm \quad 11,4\end{array}$ & $\begin{array}{r}114,6 \\
\pm 11,0\end{array}$ & $\begin{array}{r}104,1 \\
\pm \quad 11,0\end{array}$ \\
\hline $24 h$ & $\begin{array}{r}70,9 \\
+\quad 6,1\end{array}$ & $\begin{array}{r}99,5 \\
+\quad 8,9\end{array}$ & $\begin{array}{r}120,1 \\
+\quad 15,7\end{array}$ & $\begin{array}{r}97,9 \\
+\quad 2,7\end{array}$ & $\begin{array}{r}129,3 \\
\pm 16,5\end{array}$ & $\begin{array}{r}80,5 \\
+16,0\end{array}$ \\
\hline Moyenne journalière . & $\begin{array}{r}89,3^{a} \\
\pm 15,6\end{array}$ & $\begin{array}{r}114,4^{b} \\
\pm 15,8\end{array}$ & $\begin{array}{r}125,1^{\mathrm{b}} \\
\pm 14,0\end{array}$ & $\begin{array}{l}103,8^{b c} \\
\pm 12,0\end{array}$ & $\begin{array}{r}115,9^{b} \\
\pm 22,3\end{array}$ & $\begin{aligned} & 91,4^{\text {ac }} \\
\pm & 16,6\end{aligned}$ \\
\hline
\end{tabular}

Valeur moyenne pour 6 individus \pm SD. Les moyennes n'ayant pas le même indice sont significativement différentes $(P<0,01)$.

n'y a pas de réponse de croissance durant les 15 premiers jours à la nouvelle température et que la consommation alimentaire augmente lorsque les effets du stress thermique disparaissent, c'est-à-dire le $4^{\mathrm{e}}$ jour suivant la hausse de température. Nos résultats confirment leurs observations. En effet, aucune variation dans le poids des truites n'est remarquée au cours des 8 premiers jours à $17^{\circ} \mathrm{C}$ 
et un accroissement de la prise de nourriture est constaté le $5^{\mathrm{e}}$ jour, c'est-à-dire au moment où les valeurs du rapport hépatosomatique et du taux des protéines hépatiques démontrent l'existence de perturbations métaboliques importantes dans les premiers jours qui suivent le changement de température. En fait, durant cette période, il semble que les modifications, au niveau du foie, soient proches de celles qui ont été observées chez des truites gardées à une température supérieure de 10 et $12{ }^{\circ} \mathrm{C}$ à la température saisonnière : chez ces animaux, une diminution du rapport hépatosomatique a été notée (Jürss, 1979 ; Parent et Vellas, 1981).

Ammoniémie et urémie. - Les déterminations de l'ammoniémie et de l'urémie n'ont pas révélé de différences entre les moyennes journalières à $9{ }^{\circ} \mathrm{C}$ et à $17^{\circ} \mathrm{C}$. Or, d'une façon générale, une augmentation thermique provoque chez les Poissons, un accroissement des quantités d'ammoniaque et d'urée excrétées (Pora et Précup, 1960 ; Péquin et Serfaty, 1963 ; Vellas, 1973 ; Guerin-Ancey, 1976 ; Kaushik, 1980 b ; Jobling, 1981). Nos résultats indiqueraient ainsi que l'élimination des déchets azotés est beaucoup plus rapide à $17^{\circ} \mathrm{C}$ qu'à $9^{\circ} \mathrm{C}$, ce qui peut s'expliquer, en partie, par l'augmentation des fréquences cardiaques et respiratoires observées, chez les Poissons, après une élévation de température (Labat, 1966 ; Roberts, 1973 ; Heath et Hughes, 1973 ; Courtois, 1976 ; Siegmund et Vogel, 1977).

Cependant, une augmentation de l'ammoniaque plasmatique a été notée lorsque des truites arc-en-ciel vivant à $9{ }^{\circ} \mathrm{C}$ subissent une hausse thermique jusqu'à $18^{\circ} \mathrm{C}$ (Fauconneau et Luquet, 1979). Nous-mêmes, dans une étude effectuée chez des truites acclimatées à 15 et $20^{\circ} \mathrm{C}$, nous avons observé une élévation de la concentration ammoniacale et uréique du sang lorsque les animaux son maintenus à $20^{\circ} \mathrm{C}$ (résultats non encore publiés). Mais, une vérification de nos expérimentations par la recherche de l'ammoniémie et de l'urémie chez des truites gardées six semaines à $9^{\circ} \mathrm{C}$ et à $17^{\circ} \mathrm{C}, \mathrm{n}^{\prime} \mathrm{a}$ jamais permis de déceler de différences entre les deux lots de Poissons. En conséquence, toutes ces données conduisent à supposer que, chez la Truite arc-en-ciel, les processus responsables de la production et de l'élimination de l'ammoniaque et de l'urée peuvent assurer une constance des moyennes journalières de l'ammoniémie et de l'urémie au moins entre $9^{\circ} \mathrm{C}$ et $17^{\circ} \mathrm{C}$, mais qu'à partir de $18^{\circ} \mathrm{C}$, I'un des processus ou l'ensemble des processus subit une altération qui entraîne une augmentation de la teneur en ammoniaque et en urée du sang. Cette hypothèse doit, toutefois, être confirmée.

Après le repas, des modifications de l'ammoniémie sont remarquées. Ceci est en accord avec la mise en évidence, chez les Téléostéens, de variations postprandiales de l'excrétion ammoniacale (Brett et Zala, 1975; Richly et Marina, 1977 ; Kaushik, 1980 a, b ; Paulson, 1980 ; Jobling, 1981). De plus, si on observe chez la Truite arc-en-ciel, dans les premiers jours qui suivent l'élévation thermique, une évolution assez variable de la concentration ammoniacale du sang au cours du nycthémère, ce qui dénote des ajustements métaboliques à la nouvelle température, des résultats analogues ont été rapportés pour la même espèce, après une hausse de température (Fauconneau et Luquet, 1979). 
Des variations postprandiales de l'urémie sont également notées chez la Truite arc-en-ciel. Elles vérifient ainsi l'observation de fluctuations nycthémérales de l'élimination de l'urée chez ce Poisson (Kaushik, 1980 a). Après l'élévation thermique, on remarque après le repas diverses modifications de l'évolution de l'urémie comme pour l'ammoniémie, mais le fait qu'elles soient plus tardives que celles de la concentration ammoniacale du sang (elles apparaissent seulement le $2^{\mathrm{e}}$ jour à $17^{\circ} \mathrm{C}$ ) est assez difficile à expliquer.

Activités enzymatiques. - Immédiatement après la hausse thermique, on observe une élévation des activités enzymatiques et cette activation se retrouve le $23^{e}$ jour à $17^{\circ} \mathrm{C}$ pour la glutamate deshydrogénase, la glutaminase et l'uricase. Ce dernier résultat suggère qu'il n'y a pas de phénomènes de compensation à la température dans le cas des trois enzymes citées, ce qui est confirmé pour la glutamate deshydrogénase. En effet, lorsque les analyses sont effectuées à la même température, l'activité de cette enzyme est identique chez les animaux acclimatés à $9^{\circ} \mathrm{C}$ et chez les animaux maintenus 23 jours à $17^{\circ} \mathrm{C}$. Nos résultats vérifieraient ainsi les observations de Hazel et Prosser $(1970,1974)$ puisque ces chercheurs indiquent que, chez les Poecilothermes, il n'y a pas de compensation à la température dans le cas des enzymes de dégradation de métabolites et particulièrement de produits du métabolisme azoté. En outre, il est évident que l'accroissement, induit par l'élévation thermique, de l'activité de la glutamate deshydrogénase, de la glutaminase et de l'uricase s'accorde avec l'augmentation des quantités d'ammoniaque et d'urée excrétées, généralement signalée, chez les Poissons, après une hausse de température.

La réduction de l'activité de l'arginase notée le $23^{e}$ jour à $17{ }^{\circ} \mathrm{C}$, après son augmentation durant les premiers jours à ce niveau thermique, démontre, par contre, une capacité d'adaptation de l'enzyme à la température de $17^{\circ} \mathrm{C}$. D'après Marinescu (1973), chez l'Orfe (/dus idus L.) soumis à divers changements thermiques, l'adaptation de l'arginase à la température se ferait suivant le type de «compensation partielle » (Precht, 1958). Mais, nos résultats chez la Truite indiquent nettement une "compensation thermique parfaite " (Precht, 1958) puisque les moyennes journalières des valeurs enzymatiques ne sont pas différentes à $9{ }^{\circ} \mathrm{C}$ et le $23^{\mathrm{e}}$ jour à $17^{\circ} \mathrm{C}$. Par notre étude, nous ne pouvons, évidemment, connaître ou envisager les mécanismes qui en sont responsables. Toutefois, elle peut facilement s'expliquer par le fait que l'arginine est un acide aminé essentiel chez les Téléostéens (Cowey, 1979).

Une certaine similitude paraît exister, à $9{ }^{\circ} \mathrm{C}$ et le $23^{e}$ jour à $17^{\circ} \mathrm{C}$, entre l'évolution au cours du nycthémère, de l'activité glutaminasique et de l'ammoniémie, et de l'activité arginasique et de l'urémie. La glutaminase est plus active lou tend à être plus active) lorsque le taux d'ammoniaque dans le sang est le plus important ( 3 à $12 \mathrm{~h}$ après le repas à $9^{\circ} \mathrm{C}, 12 \mathrm{~h}$ après le repas à $17^{\circ} \mathrm{C}$ ) ; l'activité de l'arginase est la plus élevée lorsque la teneur en urée du sang est la plus forte (6 $\mathrm{h}$ après le repas à $9^{\circ} \mathrm{C}, 6$ et $12 \mathrm{~h}$ après le repas à $17^{\circ} \mathrm{C}$ ). II semblerait, ainsi, suivant ces résultats, que les variations nycthémérales de l'ammoniémie proviennent principalement de la dégradation de la glutamine, et celles de l'urémie de la dégradation de l'arginine. 


\section{Conclusion.}

Chez la Truite arc-en-ciel, l'augmentation de température retenue ne paraît pas influer sur les moyennes journalières de l'ammoniémie et de l'urémie, néanmoins elle entraîne des modifications de l'évolution nycthémérale de ces deux paramètres sanguins.

L'élévation thermique provoque un accroissement de l'activité de la glutamate deshydrogénase, de la glutaminase et de l'uricase, ce qui est en accord avec les variations de l'excrétion ammoniacale et uréique observées, chez les Téléostéens, après une hausse de température.

Enfin, si dans les jours qui suivent le changement thermique l'activité de l'arginase s'élève légèrement, par la suite cette enzyme manifeste une capacité d'adaptation à la nouvelle température suivant le type de " compensation parfaite ", capacité sans nul doute imputable au besoin en arginine reconnu chez les Téléostéens.

Reçu en décembre 1981. Accepté en mai 1982.

\section{Références}

BENNEMANN R., 1977. The influence of acclimation and experimental temperature on glutamate dehydrogenase of carp red lateral muscle (Cyprinus carpio L.). J. thermal Biol., 2, 209-214.

BERNT E., BERGMEYER H.U., 1974. L-glutamate UV-assay with glutamate dehydrogenase and NAD, 1704-1708. In BERGMEYER H. U., Methods of enzymatic analysis. Verlag Chemie, Weinheim ; Acad. Press, New York and London.

BRETT J. R., ZALA C. A., 1975. Daily pattern of nitrogen excretion and oxygen consumption of sockeye salmon (Oncorhynchus nerka) under controlled conditions. J. Fish. Res. Board Can., 32, 2479-2486.

CONWAY E. J., 1962. Microdiffusion analysis and volumetric error. Crosby Lockwood ed., London.

COURTOIS L. A., 1976. Respiratory responses of Gillichthys mirabilis to changes in temperature, dissolved oxygen and salinity. Comp. Biochem. Physiol., 53 A, 7-10.

COWEY C. B., 1979. Protein and amino acid requirements of finfish. In Proc. World Symp. on Finfish nutrition and fishfeed technology, Hamburg, Halver and Tiews ed., Heenemann Verlagsgesellschaft mbH, Berlin, Vol. I, 3-16.

CURTHOYS N. P., LOWRY O. H., 1973. The distribution of glutaminase isoenzymes in the various structures of the nephron in normal, acidotic and alkalotic rat kidney. J. biol. Chem., 248, $162-168$.

CVANCARÁ V. A., 1969 a. Comparative study of liver uricase activity in fresh-water Teleosts. Comp. Biochem. Physiol., 28, 725-732.

CVANCARA V. A., 1969 b. Distribution of liver allantoinase and allantoicase activity in fresh water Teleosts. Comp. Biochem. Physiol., 29, 631-638.

CVANCARA V. A., $1969 \mathrm{c}$. Studies on tissue arginase and ureogenesis in fresh water Teleosts. Comp. Biochem. Physiol., 30, 489-496.

FAUCONNEAU B., LUQUET P., 1979. Influence d'une élévation de température sur l'évolution de l'aminoacidémie et de l'ammoniémie après le repas chez la Truite arc-en-ciel (Salmo gairdneri R.). Ann. Biol. anim. Bioch. Biophys., 19, 1063-1079.

FROMM P. O., 1963. Studies on renal and extra-renal excretion in a fresh water Teleost, Salmo gairdneri. Comp. Biochem. Physiol., 10, 121-128. 
GILBOE D. D., WILLIAMS J. N. Jr, 1956 a. Evaluation of the Sakaguchi reaction for quantitative determination of arginine. Proc. Soc. exp. Biol. Med., 91, 535-536.

GILBOE D. D., WILLIAMS J. N. Jr, 1956 b. Colorimetric method for rapid determination of arginase activity. Proc. Soc. exp. Biol. Med., 91, 537-539.

GOLDSTEIN L., FORSTER R. P., 1965. The role of uricolysis in the production of urea by fishes and other aquatic vertebrates. Comp. Biochem. Physiol., 14, 567-576.

GOLDSTEIN L., FORSTER R. P., FANELLI G. M., 1964. Gill blood flow and ammonia excretion in the marine teleost, Myoxocephalus scorpius. Comp. Biochem. Physiol, 12, 489-499.

GUERIN-ANCEY O., 1976. Etudes expérimentales de l'excrétion azotée du Bar (Dicentrarchus labrax) en cours de croissance. I. Effets de la température et du poids du corps sur l'excrétion d'ammoniac et d'urée. Aquaculture, 9, 71-80.

HAZEL J., PROSSER C. L., 1970. Interpretation of inverse acclimation to temperature. $Z$. vergl. Physiologie, 67, 217-228.

HAZEL J., PROSSER C. L., 1974. Molecular mechanisms of temperature compensation in Poikilotherms. Physiol. Rev., 54, 620-677.

HEATH A. G., HUGHES G. M., 1973. Cardiovascular and respiratory changes during heat stress in rainbow trout (Salmo gairdneri). J. exp. Biol., 59, 323-338.

JOBLING M., 1981. Some effects of temperature, feeding and body weight on nitrogenous excretion in young plaice Pleuronectes platessa L. J. Fish. Biol., 18, 87-96.

JÜRSS K., 1979. Effects of temperature, salinity, and feeding on aminotransferase activiy in the liver and white muscle of rainbow trout (Salmo gairdneri Richardson). Comp. Biochem. Physiol., 64 B, 213-218.

KALCKAR H. H., 1947. Differential spectrophotometry of purine compound by means of specific enzymes. I. Determination of hydroxypurine compounds. J. biol. Chem., 167, 429-443.

KAUSHIK S. J., 1980 a. Influence of nutritional status on the daily patterns of nitrogen excretion in the carp (Cyprinus carpio L.) and the rainbow trout (Salmo gairdneri R.). Reprod. Nutr. Dévelop., 20, 1751-1765.

KAUSHIK S. J., $1980 \mathrm{~b}$. Influence of a rise in temperature on the nitrogen excretion of rainbow trout (Salmo gairdneri R.). Proc. HERS, Symp. EIFAC, Stavanger, Norvège (document photocopié).

LABAT R., 1966. Electrocardiologie chez les Poissons Téléostéens : influence de quelques facteurs écologiques. Th. Doc. Sci. Toulouse.

LOWRY O. H., ROSEBROUGH N. J., FARR A. L., RANDALL R. S., 1951. Protein measurement with the Folin phenol reagent. J. biol. Chem., 193, 265-275.

MARINESCU AL. G., 1973. Variation of the arginase activity related to the nutrition factor in the process of fish adaptation to temperature. Rev. roum. Biol-Zoo, 18, 289-294.

McBEAN R. L., NEPPEL M. J., GOLDSTEIN L., 1966. Glutamate deshydrogenase and ammonia production in the eel (Anguilla rostrata). Comp. Biochem. Physiol., 18, 909-920.

MORRISON R. F., 1976. Multivariate statistical methods. Mc Graw Hill, New York.

OGINO C., KARINO J., CHEN M., 1973. Protein nutrition in fish. Il. Determination of metabolic fecal nitrogen and endogenous nitrogen excretions of carp. Bull. jap. Soc. Sci. Fish., 39, 519523.

PARENT J. P., VELLAS F., 1981. Effets de variations thermiques chez la Truite arc-en-ciel (Salmo gairdneri, Rich.). Cah. Lab. Hydrobiol. Montereau, 11, 29-40.

PAULSON L. J., 1980. Models of ammonia excretion for brook trout (Salvelinus fontinalis) and rainbow trout (Salmo gairdneri). Can. J. Fish Aquat. Sci., 37, 1421-1425.

PÉQUIN L., 1967. Dégradation et synthèse de la glutamine chez la Carpe (Cyprinus carpio L.). Arch. Sci. physiol., 21, 193-203.

PÉQUIN L., SERFATY A., 1963. L'excrétion ammoniacale chez un Téléostéen dulcicole. Cyprinus carpio L. Comp. Biochem. Physiol., 10, 315-324.

PÉQUIN L., VELLAS F., BOUCHE G., 1969. La glutamine synthétase chez le Carpe (Cyprinus carpio L.). Arch. Sci. physiol., 23, 469-480.

PORA E. A., PRECUP O., 1960. Etude de l'excrétion chez les Poissons d'eau douce. Il. Influence de la température externe sur les processus d'excrétion chez la Carpe et le Carassin (en russe). Vopr. Iktiol. SSSR, 15, 138-147. 
PRECHT H., 1958. Concepts of the temperature adaptation of unchanging reaction systems of cold blooded animals, 50-78. In PROSSER C. L., Physiological adaptation. Am. Physiol. Soc., Washington D. C.

PRAETORIUS E., POULSEN H., 1953. Enzymatic determination of uric acid. Scand. J. clin. lab. Invest., 5, 273-282.

RYCHLY J., MARINA B. A., 1977. The ammonia excretion of trout during a 24-hour period. Aquaculture, 11, 173-178.

ROBERTS J. L., 1973. Effects of thermal stress on gill ventilation and heart rate in fish, 64-86. In CHAVIN W., Responses of fish to environmental changes. Thomas C. C., Publ., Springfield, Illinois.

SAVITZ J., 1969. Effects of temperature and body weight on endogenous nitrogen excretion in the bluegill sunfish (Lepomis macrochirus). J. Fish. Res. Board Can., 26. 1813-1821.

SIEGMUND R., VOGEL J., 1977. The dependence of heart rate and locomotor activity on water temperature in the carp (Cyprinus carpio L.). Experientia, 33, 1607-1609.

SMITH H. W., 1929. The excretion of ammonia and urea by the gills of fish. J. biol. Chem., 21, 727742.

VELLAS F., 1965. Effets de quelques facteurs écologiques sur l'activité des enzymes uricolytiques du foie de la Carpe miroir. Ann. Limn., 1, 435-442.

VELLAS F., 1973. Recherches sur l'uréogenèse chez les Téléostéens dulcicoles. Th. Doct. Sci. Toulouse.

VELLAS F., SERFATY A., 1967. Sur l'excrétion uréique de la Carpe (Cyprinus carpio L.). Arch. Sci physiol., 21, 185-192.

VELLAS F., PÉQUIN L., SERFATY A., 1969. Sur l'arginase et la $\gamma$-guanidino-butyrate ureohydrolase de la Carpe (Cyprinus carpio L.). Arch. Sci. physiol., 23, 401-405.

WALTON M. J., COWEY C. B., 1977. Aspects of ammoniogenesis in rainbow trout, Salmo gairdneri. Comp. Biochem. Physiol., 57 B, 143-149.

WARNER M. C., DIEHL S. A., TOMB A. M., 1978. Effects of dilution and temperature of analysis on blood serum values in rainbow trout, Salmo gairdneri. J. Fish. Biol., 13, 315-319. 